\title{
Complexes Based on Poly(styrenesulfonic acid) and Poly(ethylene oxide)
}

\author{
P. I. Vetosheva ${ }^{a, b}$, V. O. Bodaev ${ }^{a}$, O. A. Pyshkina ${ }^{a, *}$, E. A. Litmanovich ${ }^{a}$, and V. G. Sergeev ${ }^{a}$ \\ ${ }^{a}$ Faculty of Chemistry, Moscow State University, Moscow, 119992 Russia \\ ${ }^{b}$ Skolkovo Institute of Science and Technology, Moscow, 143026 Russia \\ *e-mail:pyshkina@genebee.msu.ru
}

Received November 2, 2020; revised December 7, 2020; accepted December 11, 2020

\begin{abstract}
Complexation in aqueous salt-free semidilute solutions of poly(styrenesulfonic acid) and poly(ethylene oxide) leading to the formation of soluble poly(styrenesulfonic acid)-poly(ethylene oxide) complexes is studied. It is shown that the interaction of the components in such complexes significantly weakens with an increase in temperature, as well as with a decrease in the poly(ethylene oxide) chain length. Using viscometry and light scattering, it is demonstrated that, in dilute aqueous and aqueous-saline solutions, no complexes are formed between poly(styrenesulfonic acid) and poly(ethylene oxide) and the system is a compatible mixture of the polymers in a common solvent.
\end{abstract}

DOI: $10.1134 / \mathrm{S} 156009042102010 \mathrm{X}$

\section{INTRODUCTION}

Recently, interpolymer complexes stabilized by bonds that differ from Coulomb bonds, such as hydrogen and ion-dipole bonds [1, 2], have been of great interest, since they can be used as $\mathrm{pH}$ - and temperature-responsive components in the development of microcapsules, nanoparticles, and controlled release hydrogels, as well as polymer electrolyte membranes [3]. Complexes stabilized by hydrogen bonds are formed between polymers having a proton donor (usually polyacids) and a nonionic polymer containing a strong hydrogen acceptor in the backbone. The following groups can be distinguished among water-soluble nonionic polymers studied from the point of view of complexation with polyacids: polymers containing ether groups in the main chain (e.g., PEO, PPO) or side substituents (poly(vinyl methyl ether)); polymers containing lactam groups, such as polyvinylpyrrolidone and polyvinylcaprolactam; acrylic polymers (polyacrylamide, polyisopropylacrylamide); polymeric alcohols (PVA); other synthetic polymers, such as poly(ethyloxazoline) and poly( $N$-acetyliminoethylene); and polysaccharides [4-11]. In addition, the examples of hydrogen donors are mainly limited to various poly(carboxylic acids) with the exception of those containing polyphenol, polyalcohols, and polyaniline $[12,13]$.

The formation of complexes based on polyacids is possible only in acidic media $(\mathrm{pH}<7)$. This is due to the following reasons: the acid must be protonated to form a hydrogen bond; electrostatic repulsion between deprotonated acid residues destabilizes interpolymer complexes (IPCs). Sulfonic acids, unlike carboxylic acids, are very strong and completely dissociate even in the presence of traces of water. However, in accordance with [14], stoichiometric water-insoluble complexes can be formed between some poly(sulfonic acids) and PEO in dilute aqueous solutions. The formation of a complex between PEO and poly(styrenesulfonic acid), as well as fluorinated poly(sulfonic acid) (trade name Nafion), was experimentally confirmed by turbidity measurements and IR spectroscopy. It was shown that PEO forms complexes with poly(styrenesulfonic acid) of different molecular weights at $\mathrm{pH}<0.5$ [14]. This unexpected interaction is explained by the strength of the hydrogen bond between the sulfo group and the ether, which stabilizes the IPC by shifting the acid-base equilibrium.

It is known that the properties of complexes formed via hydrogen bonding can be controlled by insignificant changes in the initial parameters and that complexation entails a significant change in the hydrodynamic properties of solutions; this makes such systems interesting for use in solving many practical problems [15-17].

In this work, we studied complexation in aqueous solutions of PSSA and PEO in different concentration modes and at different ionic strength of the solutions and molecular weight of PEO (PEG).

\section{EXPERIMENTAL}

Poly(4-styrenesulfonic acid) (PSSA, $M_{\mathrm{w}}=7.5 \times 10^{4}$, Sigma Aldrich), poly(ethylene oxide) (PEO, $M_{\mathrm{w}}=$ 
40000, Aldrich), and poly(ethylene glycol) (PEG, $M_{\mathrm{w}}=2000$, Aldrich) were used without preliminary purification. Solutions were prepared using bidistilled water deionized with a MilliQ ion exchange column system (Millipore, United States). Saline solutions were prepared using sodium chloride (Khimreaktiv, Russia).

The $\mathrm{PEO}_{40000}$ solution with a given weight concentration was prepared by dissolving a weighed portion for a day in the calculated volume of water or $0.1 \mathrm{M}$ $\mathrm{NaCl}$ solution. An aqueous solution of PSSA of a given concentration was prepared by diluting commercial $18 \mathrm{wt} \%$ solution. To prepare a PSSA solution in $0.1 \mathrm{M} \mathrm{NaCl}$, commercial $18 \mathrm{wt} \%$ solution was first diluted two times with $0.2 \mathrm{M} \mathrm{NaCl}$ solution and then $0.1 \mathrm{M} \mathrm{NaCl}$ was added to the resulting solution to reach the specified concentration. The aqueous solution of $\mathrm{PEG}_{2000}$ of a given weight concentration was prepared by dissolving a weighed portion for a day in the calculated volume of water. The aqueous solution of PSSA of a given concentration was prepared by diluting commercial $18 \mathrm{wt} \%$ solution.

The aqueous solutions of PSSA-PEO ${ }_{40000}$ with a given ratio $Z=\left(n\left(\mathrm{PEO}_{40000}\right)\right) /\left(n\left(\mathrm{PEO}_{40000}\right)+\right.$ $n$ (PSSA) $)(Z=0,0.32,0.59,0.81,0.91,0.98,1.0)$ were prepared by mixing different volumes of $\mathrm{PEO}_{40000}$ and PSSA aqueous solutions of the same weight concentration at the intrinsic $\mathrm{pH}$ values of the initial components. The aqueous solutions of PSSA-PEG 2000 were prepared in a similar way. The saline solutions of complexes and mixtures were prepared by mixing $\mathrm{PEO}_{40000}$ and PSSA solutions in $0.1 \mathrm{M} \mathrm{NaCl}$.

The relative viscosity of solutions $\eta_{\text {rel }}$ was determined in an Ubbelohde capillary viscometer; the solvent efflux time was $t_{0}=47 \mathrm{~s}\left(25^{\circ} \mathrm{C}\right)$. Before measurements the samples were thermostatted for $15 \mathrm{~min}$; the temperature in the cell was maintained with an accuracy of $\pm 0.2^{\circ} \mathrm{C}$.

The dynamic viscosity was calculated as $\eta=\frac{t \eta_{0}}{t_{0}}$, where $t$ is the solution flow time and $\eta_{0}$ is the viscosity of the solvent (water) at a given temperature.

Specific $\eta_{\text {sp }}$ and reduced viscosity $\eta_{\text {red }}$ were calculated by the formulas

$$
\eta_{\mathrm{sp}}=\eta_{\text {rel }}-1, \quad \eta_{\text {red }}=\frac{\eta_{\text {rel }}-1}{C}
$$

( $C$ is the solution concentration, $\mathrm{g} / \mathrm{dL}$ ).

Static and dynamic light scattering were measured on a Photocor Complex spectrometer (Photocor Instruments, Russia) equipped with a $\mathrm{He}-\mathrm{Ne}$ laser with a power of $10 \mathrm{mV}(\lambda=633 \mathrm{~nm})$. The solutions under study were preliminarily filtered twice through Durapore ${ }^{\circledR}$ filters (Millipore, United States, hydrophilized Teflon, pore diameter of $0.22 \mu \mathrm{m})$. The scattering intensity in static scattering experiments was averaged over ten independent measurements. The molecular weights of PSSA, PEO, and their mixture were determined at a scattering angle of $90^{\circ}$; the results were processed using the Debye equation

$$
K C / R_{\Theta}=1 / M_{W}+2 A_{2} C,
$$

where the optical constant of the solution $K=$ $\frac{4 \pi^{2} n^{2}\left(\frac{\partial n}{\partial c}\right)^{2}}{\lambda_{0}^{4} N_{\mathrm{A}}}, R_{\Theta}$ is the Rayleigh ratio, $C$ is concentration, and $A_{2}$ is the second virial coefficient. The error in determining molecular weights did not exceed $5 \%$.

The refractive index increments of solutions $\partial n / \partial c$ were determined using an Optilab T-Rex differential refractometer (Wyatt, United States) at $\lambda=633 \mathrm{~nm}$; the error was $0.4-1.1 \%$.

The correlation functions of scattered light intensity fluctuations in dynamic light scattering studies were measured using a Photocor-FC 288-channel correlator with a pseudocross-correlation photon counting system (Photocor Instruments, Russia). The data were processed with the DynaLS software. The hydrodynamic radii of macromolecules were calculated from the diffusion coefficients using the Einstein-Stokes equation

$$
R_{\mathrm{h}}=\frac{k T}{6 \pi \eta_{0} D}
$$

where $k$ is the Boltzmann constant, $\eta_{0}$ is the viscosity of the solvent, and $D$ is the diffusion coefficient.

\section{RESULTS AND DISCUSSION}

It is known that the structure and properties of solutions of interpolymer complexes in most cases depend on the concentration mode in which they are determined [18]. In dilute solutions, such complexes usually form individual particles, while in semidilute solutions, intermolecular aggregates forming the network of entanglements are produced. Therefore, before studying complexation in the PSSA-PEO 40000 system, first the boundaries of the concentration modes of individual components PSSA and $\mathrm{PEO}_{40000}$ and the mixture of composition $Z=0.81$ were estimated. The boundaries of concentration modes in solutions of the initial polymers and their mixture were determined by capillary viscometry (Figs. 1, 2). The solutions of polymer and their mixture were prepared in water in the absence of a low molecular weight electrolyte. PEO is a nonionic polymer, and PSSA is a negatively charged polyelectrolyte under these conditions.

The concentration dependence of specific viscosity plotted in logarithmic coordinates for $\mathrm{PEO}_{40000}$ (Fig. 1, curve 1) consists of two linear sections. The initial section $(C<3.6 \%)$ has an angular slope of 1.2. This means that, in this concentration range, $\eta_{\mathrm{sp}} \propto$ 


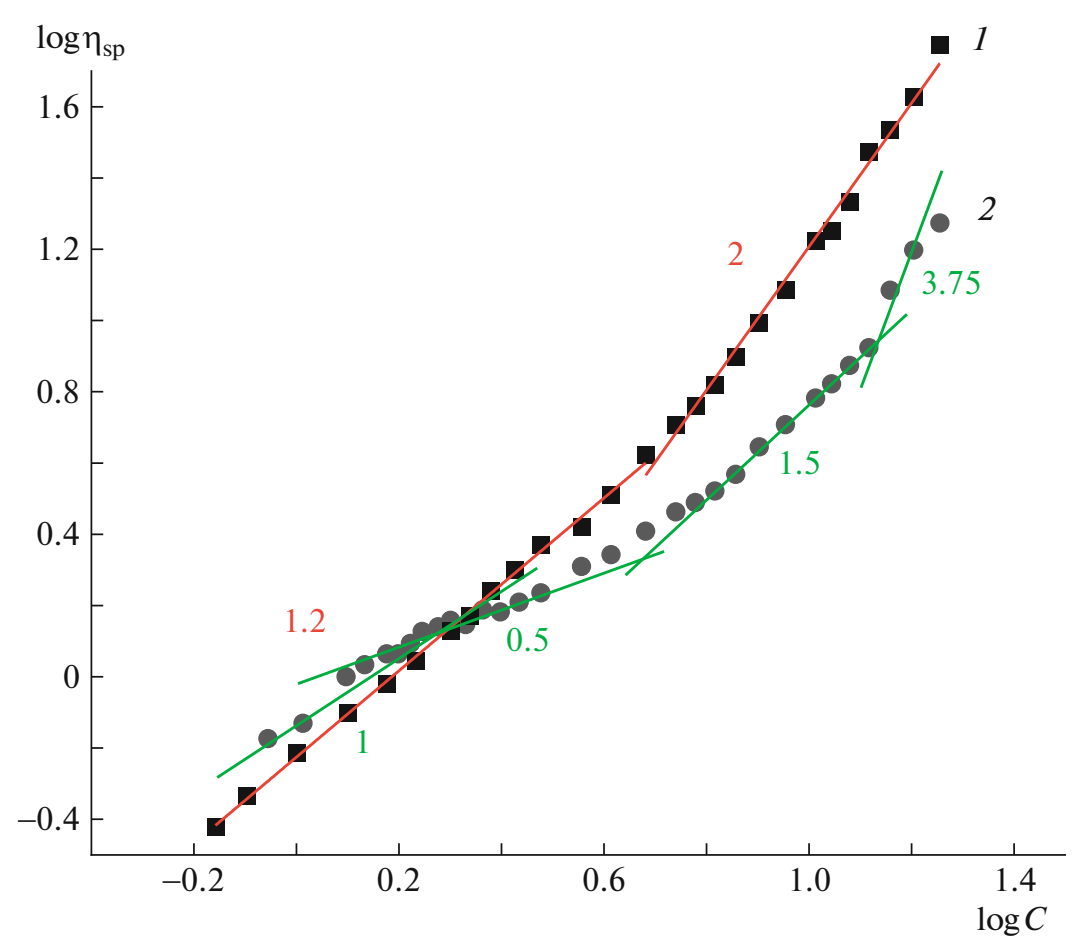

Fig. 1. Log specific viscosity of (1) $\mathrm{PEO}_{40000}$ and (2) PSSA vs. log concentration. Color drawings can be viewed in the electronic version.

$C^{1.2}$, which, according to the scaling model [19], corresponds to a dilute solution of a nonionic polymer. The concentration at which the slope of the dependence changes corresponds to the crossover concentration $C^{*}$ (Table 1). The angular slope of the second linear section $(3.6<C \leq 18 \%)$ is two. The scaling ratio $\eta_{\mathrm{sp}} \propto C^{2}$ corresponds to a semidilute solution mode

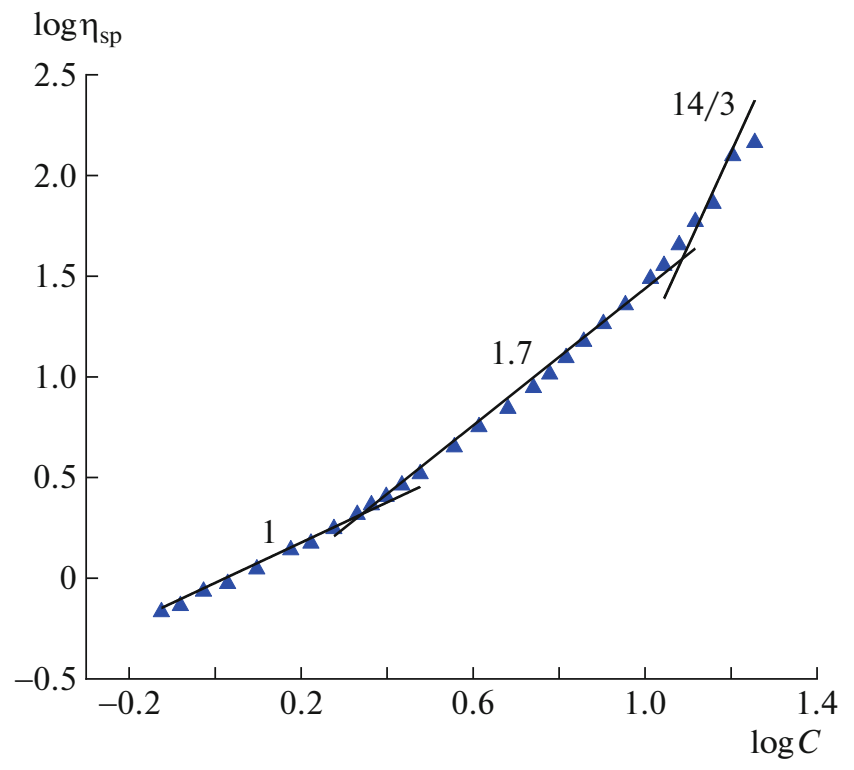

Fig. 2. Log specific viscosity of the PSSA-PEO 40000 mixture $(Z=0.81)$ vs. $\log$ concentration. without the network of entanglements for an uncharged polymer. It is important to note that, in the studied concentration range up to $C=18 \%$, no network of entanglements is formed in $\mathrm{PEO}_{40000}$ solutions.

The concentration dependence of the specific viscosity of PSSA solutions (Fig. 1, curve 2) consists of four sections and fully corresponds to the scaling description of polyelectrolyte solutions [20, 21]. In the low concentration range $(C<1.8 \%), \eta_{\mathrm{sp}} \propto C^{1}$, which is typical of a dilute polyelectrolyte solution in the presence of a salt. Even through the experiment was carried out without addition of a low molecular weight electrolyte, the slight content of residual salts in the initial PSSA solution creates a nonzero ionic strength of the solution. The effect manifests itself only at very low concentrations of the polyelectrolyte; with an increase in polyelectrolyte concentration the contribution of its own counterions neutralizes it. In the second section $(1.8<C<6.6 \%), \eta_{\mathrm{sp}} \propto C^{0.5}$; this exponent coincides with the values of the scaling model for a semidilute polyelectrolyte solution without the network of entanglements. The change in the angular slope after this section of the curve corresponds to the concentration of the formation of entanglements $C_{\mathrm{e}}$. In the concentration range $6.6<C<12 \%, \eta_{\mathrm{sp}} \propto C^{1.5}$, which corresponds to a semidilute mode with the network of entanglements. In the high concentration range $(>13 \%), \eta_{\text {sp }} \propto C^{3.75}$, which, according to the scaling model, corresponds to the semidilute mode of the polyelectrolyte solution with the network of entan- 
Table 1. Concentration mode boundaries for $\mathrm{PEO}_{40000}$, PSSA, and the PSSA-PEO ${ }_{40000}$ mixture $(Z=0.81)$

\begin{tabular}{l|c|c|c}
\hline \multicolumn{1}{c|}{ Sample } & $C^{*}$, wt $\%$ & $C_{\mathrm{e}}, \mathrm{wt} \%$ & $C_{\mathrm{d}}, \mathrm{wt} \%$ \\
\hline PEO $_{40000}$ & 3.6 & $>18$ & - \\
PSSA & 1.3 & 6.6 & 13 \\
PSSA-PEO $_{40000}$ & 1.1 & 2.3 & 12 \\
\hline
\end{tabular}

Concentrations $C<C^{*}$ correspond to the dilute solution mode; in the range of $C^{*}<C<C_{\mathrm{e}}$, there is the semidilute solution mode without entanglements; and at $C>C_{\mathrm{e}}$, the semidilute mode with the network of entanglements is realized.

glements in a good solvent under conditions of complete suppression of electrostatic interactions.

The dependence obtained for a solution of PSSA$\mathrm{PEO}_{40000}$ mixture with $Z=0.81$ (Fig. 2) differs from those obtained for both $\mathrm{PEO}_{40000}$ and PSSA. The initial section $(C<2.3 \%)$ has the same angular slope as the PSSA dependence, that is, corresponds to a dilute polyelectrolyte solution at a nonzero ionic strength. The section with a slope of 0.5 corresponding to the semidilute mode for polyelectrolytes without the network of entanglements disappears, which indicates that interaction between PSSA and $\mathrm{PEO}_{40000}$ is possible. In the second section $(2.3<C<12 \%)$, the angular slope is 1.7 ; this concentration range can be interpreted as a semidilute mode for a polyelectrolyte with the network of entanglements. In the high concentration range $(C>12 \%), \eta_{\mathrm{sp}} \propto C^{14 / 3}$, which corresponds to a semidilute solution of a polyelectrolyte with the

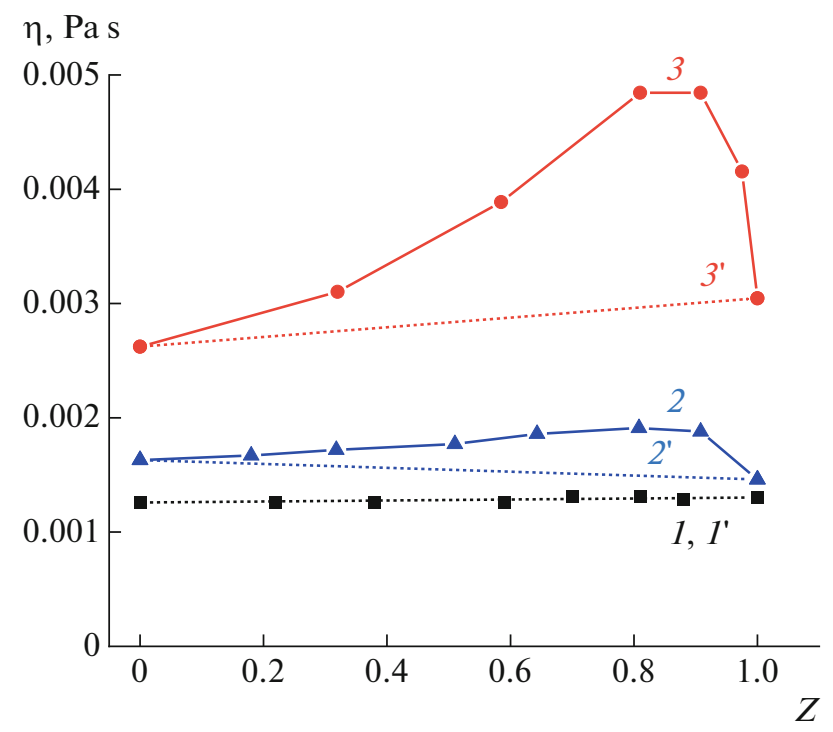

Fig. 3. Dependences of the dynamic viscosity of PSSA$\mathrm{PEO}_{40000}$ mixtures at a concentration of (1) 0.5 , (2) 1 , and (3) $3 \%$ and the corresponding dependences of the additive viscosity of PSSA-PEO ${ }_{40000}$ mixtures at a concentration of ( $\left.I^{\prime}\right) 0.5,\left(2^{\prime}\right) 1$, and ( $\left.3^{\prime}\right) 3 \%$ on mixture composition $Z$. network of entanglements with complete screening of electrostatic interactions in the $\theta$ solvent. The boundaries of concentration modes are presented in Table 1 .

When two polymers of different chemical nature are mixed in a common solvent, one of three options is realized, namely, compatibility (the system is a mixture of noninteracting components in which no phase separation is observed), incompatibility (accompanied by separation into phases, each of which is enriched in one of the polymers), or interaction (in this case, an interpolymer complex is formed in solution). For complexes of poly(acrylic acid) with PEO, poly(vinyl caprolactam), poly(vinyl glycol ethers), and cellulose ethers $[6,22,23]$ in dilute solutions, the value of $\mathrm{pH}_{\mathrm{cr}}$ below which the IPC precipitates from solution was determined. Above $\mathrm{pH}_{\mathrm{cr}}$, the complex exists in solution in the form of hydrophilic soluble associates. At $\mathrm{pH}>\mathrm{pH}_{\mathrm{cr}}$, the viscosity of the solution increases sharply owing to destruction of the compact structure of the IPC. Sometimes the value of $\mathrm{pH}_{\mathrm{cr} 2}$ is distinguished above which the complex is destroyed and the polymers coexist in solution as individual components. Then in the range of $\mathrm{pH}<\mathrm{pH}_{\mathrm{cr} 1}$, the interpolymer complex exists in the form of compact hydrophobic aggregates; in the range of $\mathrm{pH}_{\mathrm{cr} 1}<\mathrm{pH}<$ $\mathrm{pH}_{\mathrm{cr} 2}$, the interpolymer complex exists in the form of hydrophilic associates; in the range of $\mathrm{pH}>\mathrm{pH}_{\mathrm{cr} 2}$, no interaction occurs. Thus, the interpolymer complex (the classical IPC) is formed by the cooperative system of hydrogen bonds which facilitates compaction of the volume of interacting (interpenetrating) coils of complementary macromolecules (the model of a zipper with defects). The hydrophilic associate is formed with the participation of a much smaller number of hydrogen bonds than the IPC, while PAA macromolecules included in the associate have a higher degree of ionization than PAA macromolecules that form the conventional IPC. The formation of an interpolymer hydrophilic associate is not accompanied by the compaction of interacting macrocoils.

It should be noted that the solutions of PSSA$\mathrm{PEO}_{40000}$, PSSA-PEG 2000 , and $\mathrm{PSSNa}-\mathrm{PEO}_{40000}$ mixtures in the entire studied concentration and temperature range remain transparent at any ratio of polymer components. This means that incompatibility does not manifest itself in the systems under study.

To ascertain whether the interaction between polymers in solution occurs, the method of viscometry was used. In the absence of interaction, the viscosity of the mixture should be additively composed of the viscosities of the components of the corresponding concentration. We first explored dilute solutions of the initial components and mixtures. Figure 3 (curve 1, squares) shows the dependence of the dynamic viscosity of PSSA-PEO 40000 mixtures at a concentration of 0.5 wt $\%$. The value $Z=0$ corresponds to a $0.5 \%$ PSSA solution, while $Z=1-0.5 \%$ corresponds to a PEO solution. For comparison, the same figure shows the 
dependence of the additive viscosity on the mixture composition calculated under the assumption of the absence of interactions according to the algorithm from [24] (Fig. 3, curve 1').

As can be seen, the dynamic viscosity of the mixture (Fig. 3, curve 1) is almost the same as the additive value (Fig. 3, curve 1 '), which indicates the absence of interaction of two polymers at a concentration of $0.5 \%$, that is, in the dilute solution mode. With an increase in concentration to $1 \mathrm{wt} \%$ (Fig. 3, curve 2), which is close to the crossover concentration for the PSSA-PEO ${ }_{40000}$ mixture of composition $Z=0.8$, there is a slight positive deviation from additivity. This suggests the appearance of interaction between the components of the mixture accompanied by the formation of soluble complexes. And, as follows from
Fig. 3 (curve 3), in semidilute solutions (3\%), the dynamic viscosity of the mixture increases significantly with increasing $Z$ from zero to $\sim 0.8$, remains almost constant with increasing $Z$ to 0.9 , and noticeably decreases with further increase in $Z$ to 1 . In other words, there is a significant positive deviation from additivity (Fig. 3, curve 3'), which indicates the interaction of the two polymers in the system. The greatest deviation from additivity is observed at mixture compositions $Z=0.8-0.9$, which corresponds to the maximum degree of binding of polymer components. Such an interaction can be implemented by means of complexation between PSSA and $\mathrm{PEO}_{40000}$ during the formation of hydrogen bonds between the oxygen atom in the PEO chain and the hydrogen atom in the sulfo group of the PSSA chain:

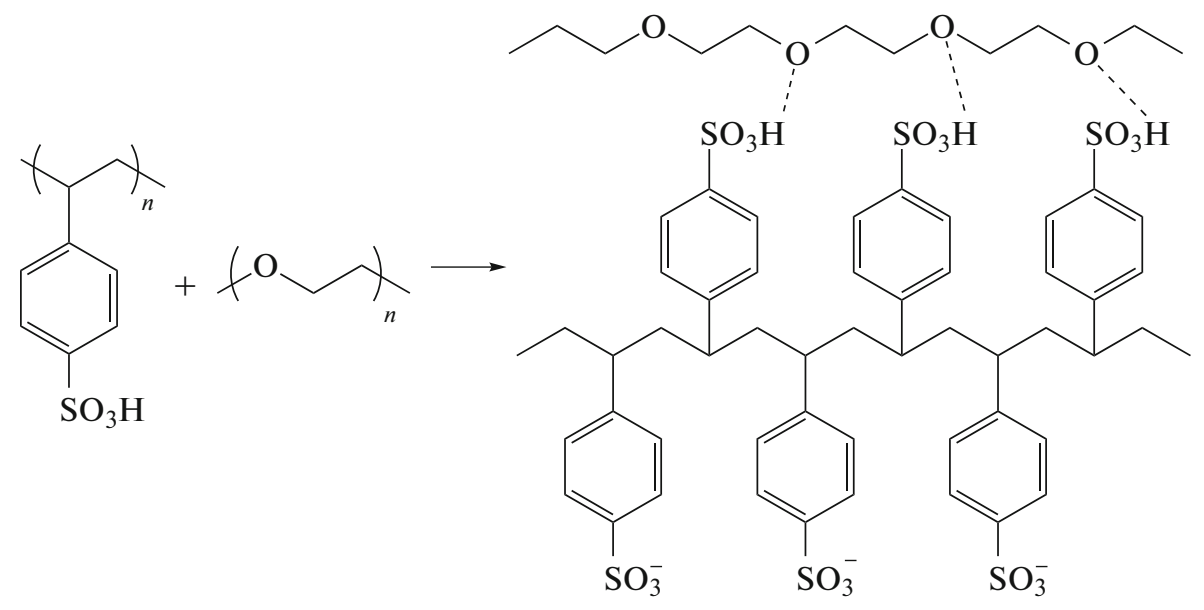

The formation of complexes between PSSA and PEO was recently described in [14]. However, these studies were carried out only in a strongly acidic medium $(\mathrm{pH}<0.5)$ with the additional acidification of aqueous solutions with $\mathrm{HCl}$ and only for stoichiometric water-insoluble complexes. In this work, for the first time, we studied in detail the aqueous solutions of PSSA and PEO in the absence of additional acidifying agents $(\mathrm{pH} \sim 2)$.

Despite the fact that PSSA belongs to the class of strong polyelectrolytes, a significant part of sulfo groups in aqueous solutions remain unionized owing to the effect of counterion condensation [25]. It is these undissociated groups that are capable of forming hydrogen bonds with the oxygen atoms of PEO, which leads to the formation of complexes. The composition of mixtures in the composition range $Z \approx 0.8$ corresponds to the molar ratio of units [PEO] : [PSSA] $\approx$ $4: 1$. Considering the degree of polymerization of $\mathrm{PEO}_{40000}(\approx 900)$ and PSSA $(\approx 400)$, one can assume that, at such a composition $Z$, there are, on average, two $\mathrm{PEO}_{40000}$ macromolecules per PSSA macromolecule. Surplus $\mathrm{PEO}_{40000}$ units and ionized PSSA regions provide the solubility of the complex. The structure of the PSSA-PEO complex with maximum interactions is schematically shown below.<smiles>CCCOCCOCCOc1ccc(C(CC(CC(CC)c2ccc(S(=O)(=O)O)cc2)c2ccc(S(=O)(=O)OCCOCC)cc2)CC(CC(CC(CC(CC)c2ccc(S(=O)(=O)O)cc2)c2ccc(S(=O)(=O)O)cc2)c2ccc(S(=O)(=O)O)cc2)c2ccc(S(=O)(=O)OCCOCC)cc2)cc1</smiles>

The initial components PSSA and $\mathrm{PEO}_{40000}$ and their mixture of composition $Z=0.8$ were studied by 


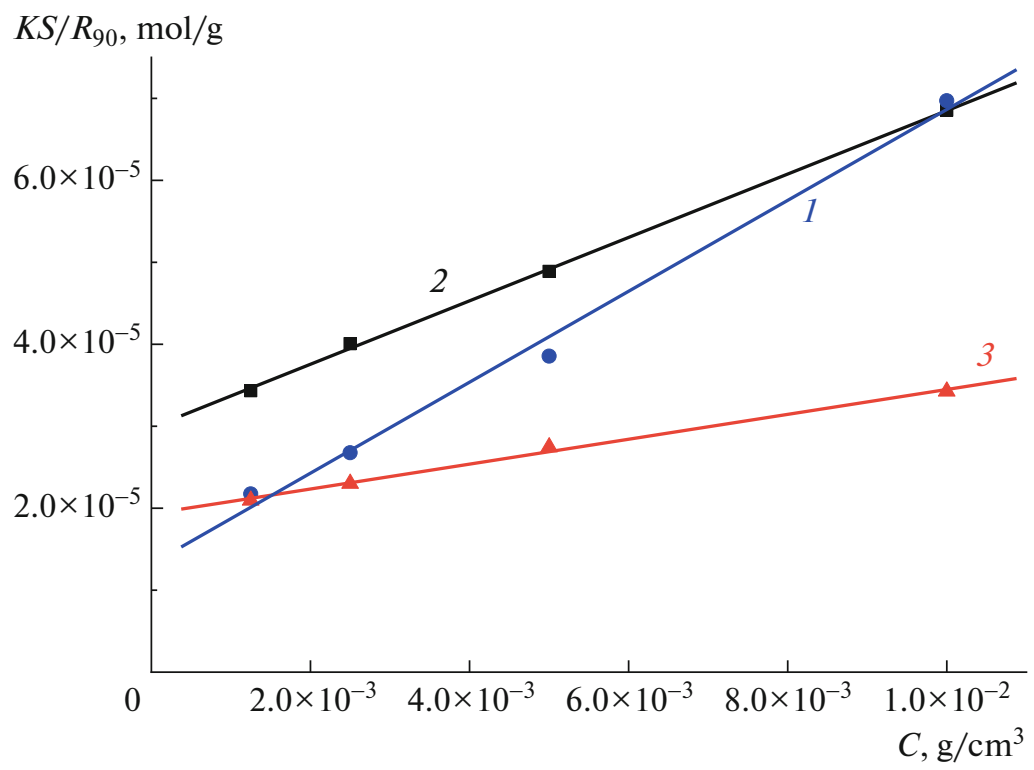

Fig. 4. Static light scattering data for solutions of (1) PSSA, (2) $\mathrm{PEO}_{40} 000$, and (3) $\mathrm{PSSA}_{-} \mathrm{PEO}_{40000}$ mixture. $Z=0.81$.

static light scattering (Fig. 4). All solutions were prepared in $0.1 \mathrm{M} \mathrm{NaCl}$, which made it possible to carry out measurements under isoionic dilution conditions. The concentrations of the working solutions were in the range of $0.125-1 \mathrm{wt} \%$. All three dependences plotted in Debye coordinates $\left(K S / R_{90}\right.$ on $\left.C\right)$ are straight lines in the studied concentration range. This makes it possible to determine the weight-average molecular weights and second virial coefficients of solutions (Table 2).

To clarify whether the PSSA-PEO ${ }_{40000}$ complex exists in dilute aqueous-saline solutions (concentration, $0.125-1 \mathrm{wt} \%$; $\mathrm{NaCl}$ concentration, $0.1 \mathrm{~mol} / \mathrm{L}$ ), it was necessary to calculate the $M_{\mathrm{w}}$ value for the mixture under the assumption that there are no interactions and to compare the obtained value with the measurement result. The mixture of composition $Z=0.81$ contains equal weight fractions of PSSA and $\mathrm{PEO}_{40000}$; hence, in the absence of interactions, $M_{\mathrm{w}}$ can be calculated as

$$
\begin{gathered}
M_{\mathrm{w}(\text { calcd })}=\sum \omega_{i} M_{i} \\
=\omega_{\mathrm{PEO}} \cdot M_{\mathrm{PEO}}+\omega_{\mathrm{PSSA}} \cdot M_{\mathrm{PSSA}} \\
M_{\mathrm{w}(\text { calcd })}=\frac{1}{2}(3.36+7.58) \times 10^{4}=5.47 \times 10^{4} .
\end{gathered}
$$

Deviation of the calculated value from the measured one $\frac{\Delta M}{M}=5 \%$ does not exceed the error of the static light scattering method. The refractive index increment $\frac{\partial n}{\partial c}$ is also additively composed of the refractive index increments of components. Consequently, in the concentration range of $1 \%$ or lower in an aqueous-saline medium, the complex is not formed and the system is a mixture of two polymers in the common solvent.

The absence of interaction in the dilute aqueoussaline solution of the PSSA- $\mathrm{PEO}_{40000}$ mixture at a concentration of $1 \%$ was confirmed by dynamic light scattering. Below are the effective hydrodynamic radii of coils in solutions of the initial polymers and their mixtures: $R_{\mathrm{h}}=6 \pm 1$ for $\mathrm{PEO}_{40000}$ and PSSA and $7 \pm 1$ for PSSA-PEO ${ }_{40000}$. The size of the coils in the mixture coincides with the sizes of the individual components within the limits of error of the method.

Thus, using viscometry and dynamic and static light scattering, it is shown that there is no interaction in the PSSA-PEO ${ }_{40000}$ system in the dilute solution mode in either an aqueous or an aqueous-saline medium.

From the data obtained for PAA-PEO complexes stabilized by hydrogen bonds, it is known that the intensity of interaction in such complexes is determined by the length of the PEO chain [26]: the degree of binding decreases significantly with a decrease in the molecular weight of PEO up to the complete absence of complexation. Therefore, it was necessary

Table 2. Static light scattering data for $\mathrm{PEO}_{40000}$, PSSA, and PSSA-PEO $_{40000}(Z=0.81)$

\begin{tabular}{l|c|c|c}
\hline \multicolumn{1}{c|}{ Sample } & $M_{\mathrm{w}} \times 10^{-4}$ & $\begin{array}{c}A_{2}, \\
\mathrm{~mol} \mathrm{~cm} / \mathrm{g}^{2}\end{array}$ & $\frac{\partial n}{\partial c}, \mathrm{~cm}^{3} / \mathrm{g}$ \\
\hline PEO $_{40000}$ & 3.36 & $1.93 \times 10^{-3}$ & 0.1323 \\
PSSA & 7.58 & $2.80 \times 10^{-3}$ & 0.2019 \\
PSSA-PEO $_{40000}$ & 5.18 & $7.59 \times 10^{-4}$ & 0.1677 \\
\hline
\end{tabular}




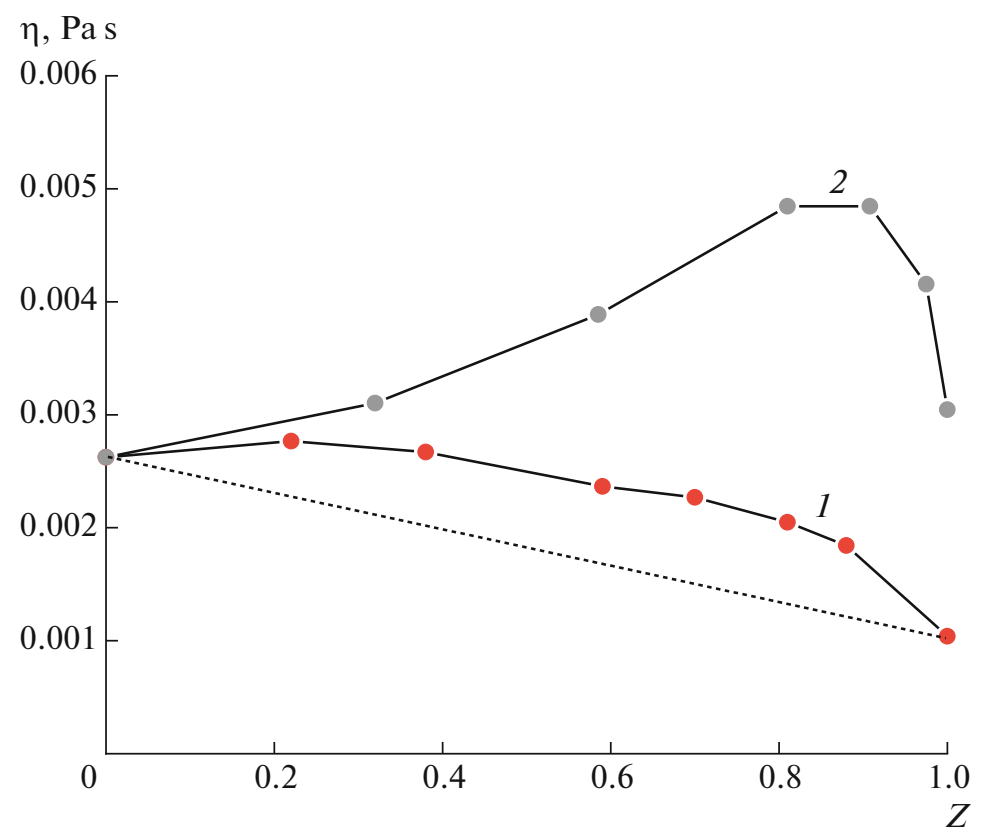

Fig. 5. (1) Dependence of the dynamic viscosity of PSSA-PEG 2000 mixtures in comparison with (2) the similar dependence for PSSA-PEO 40000 mixtures and the dependence of the additive viscosity of PSSA-PEG 2000 mixtures on mixture composition $Z$.
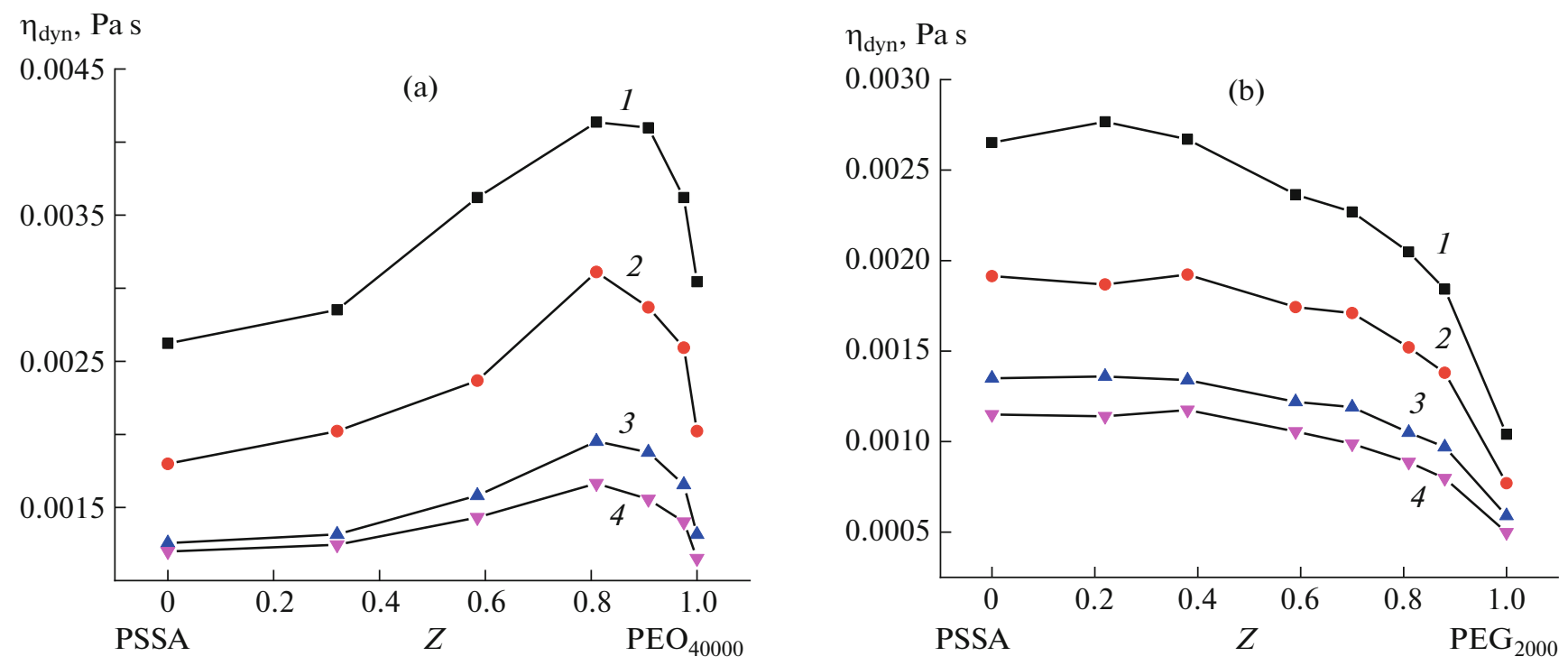

Fig. 6. Viscosity of (a) PSSA-PEO 40000 and (b) PSSA-PEG 2000 mixtures vs. composition at (1) 25, (2) 35 , (3) 50, and (4) $60^{\circ} \mathrm{C}$.

to ascertain whether the formation of complexes of PSSA with short PEO chains occurs in semidilute solutions. Figure 5 shows the dependence of the dynamic viscosity of PSSA-PEG 2000 mixtures on composition $Z$ in comparison with a similar dependence for PSSA and $\mathrm{PEO}_{40000}$.

As is seen from the dependence shown in Fig. 5 (curve 1), for all PSSA-PEG 2000 mixtures, as for PSSA-PEO ${ }_{40000}$ mixtures, there is a positive deviation of the dynamic viscosity from additivity, but this deviation is less pronounced in comparison with the PSSA-PEO 40000 system. This fact indicates that the interactions are weakened but the complexation between PSSA and short PEG $_{2000}$ persists. The relatively small deviation from additivity does not allow determining the composition of the mixture corresponding to the maximum binding in the PSSAshort-chain PEO system. 
It is known that hydrogen bonds weaken with increasing temperature [5]; therefore, the dependences of the dynamic viscosity on the mixture composition were measured for the PSSA-PEO 40000 and PSSA-PEG ${ }_{2000}$ complexes at different temperatures in the range of $25-60^{\circ} \mathrm{C}$ (Fig. 6).

For both complexes with increasing temperature, the positive deviation from additivity persists, although it becomes less pronounced. This means that heating to $60^{\circ} \mathrm{C}$ does not lead to complete destruction of the complexes despite the fact that the interactions become weaker.

The formation and structure of PSSA-PEO complexes in different concentration modes are schematically shown below.

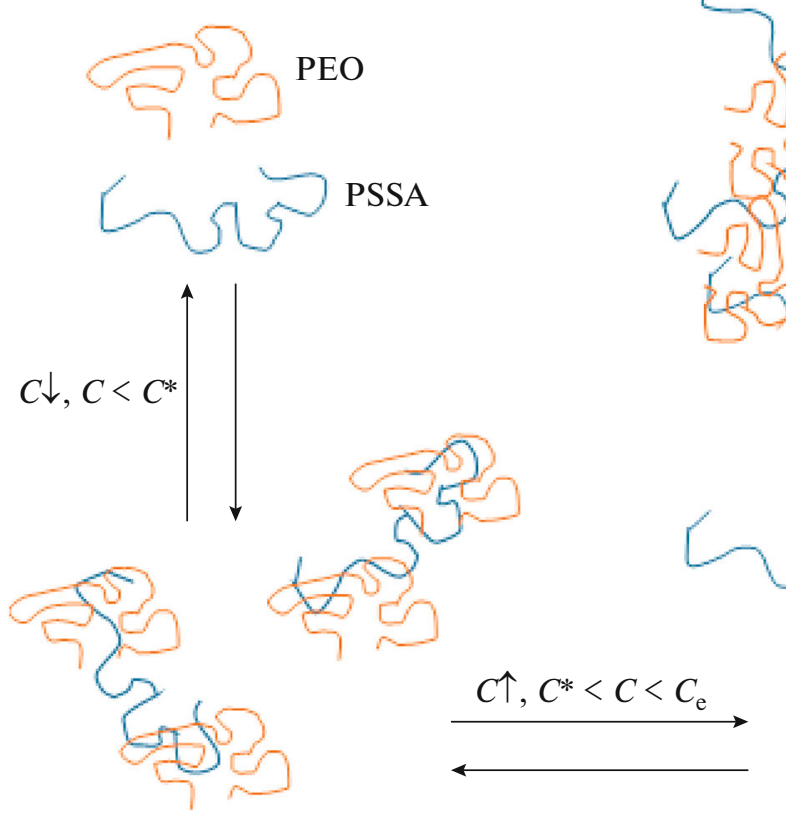

\section{CONCLUSIONS}

Thus, mixing PSSA with PEO in aqueous solutions leads to the formation of soluble complexes and the maximum binding is observed at a fourfold molar excess of PEO. The interaction of the components significantly weakens with increasing temperature and with decreasing the PEO chain length. In the semidilute solution mode, the complex is a network in which PEO chains hydrogen-bonded to neighboring PSSA coils serve as junctions. With a decrease in concentration, the dissociation of the complex occurs, and in dilute aqueous and aqueous-saline solutions, the system is a compatible mixture of the polymers in the common solvent. The results obtained suggest that a similar interaction with other nonionic polymers, such as poly(vinyl alcohol), polyvinylpyrrolidone, polyacrylamide, and cellulose ethers, is feasible.

\section{FUNDING}

This work was carried out within the framework of the project Modern Problems of Chemistry and Physical Chemistry of Macromolecular Compounds (State contract, registration number AAAA-A16-116031050014-6).

\section{OPEN ACCESS}

This article is licensed under a Creative Commons Attribution 4.0 International License, which permits use, sharing, adaptation, distribution and reproduction in any medium or format, as long as you give appropriate credit to the original author(s) and the source, provide a link to the Creative Commons license, and indicate if changes were made. The images or other third party material in this article are included in the article's Creative Commons license, unless indicated otherwise in a credit line to the material. If material is not included in the article's Creative Commons license and your intended use is not permitted by statutory regulation or exceeds the 
permitted use, you will need to obtain permission directly from the copyright holder. To view a copy of this license, visit http://creativecommons.org/licenses/by/4.0/.

\section{REFERENCES}

1. V. V. Khutoryanskiy, Int. J. Pharm. 334, 15 (2007).

2. V. Soldi, H. M. Wilhelm, M. D. S. Soldi, J. R. S. Rodrigues, A. T. N. Pires, and M. R. Sierakowski, Polym. Int. 49, 81 (2000).

3. Y.-S. Ye, J. Rick, and B.-J. Hwang, Polymers 4, 913 (2012).

4. K. L. Smith, A. E. Winslow, and D. E. Petersen, Ind. Eng. Chem. 51, 1361 (1959).

5. F. E. Bailey, Jr., R. D. Lundberg, and R. W. Callard, J. Polym. Sci., Part A 2, 845 (1964).

6. E. M. Shaikhutdinov and G. A. Mun, Vestn. KazNU, Ser. Khim. 65, 445 (2012).

7. Z. S. Nurkeeva, G. A. Mun, and V. V. Khutoryanskij, Polym. Sci., Ser. B 43, 146 (2010).

8. V. V. Khutoryanskiy, G. A. Mun, Z. S. Nurkeeva, and A. V. Dubolazov, Polym. Int. 53, 1382 (2004).

9. Y. Wang, J. He, S. Aktas, S. A. Sukhishvili, and D. Kalyon, J. Rheol. 61, 1103 (2019).

10. Y. Matsumura and K. Iwai, Polymers 11, 1196 (2019).

11. V. V. Khutoryanskiy and G. Staikos, Hydrogen-Bonded Interpolymer Complexes: Formation, Structure and Applications (World Sci., Singapore, 2009).

12. I. Erel, H. Schlaad, and A. L. Demirel, J. Colloid Interface Sci. 361, 477 (2011).
13. S. Ma, Q. Yuan, X. Zhang, S. Yang, and J. Xu, Colloids Surf., A 471, 11 (2015).

14. L. Maldonado, G. Debais, F. Davia, L. P. M. De Leo, and M. Tagliazucchi, Soft Matter 15, 9318 (2019).

15. C.-Yi. Tsai, C.-H. Chung, and J.-L. Hong, ACS Omega 3, 4423 (2018).

16. K. M. Hutchins, Roy. Soc. Open Sci. 5, 180564 (2018).

17. Y. Wang and S. A. Sukhishvili, Soft Matter 12, 8744 (2016).

18. E. A. Litmanovich, E. V. Kotova, and V. V. Efremov, Colloid Polym. Sci. 297, 371 (2019).

19. M. Rubinstein and R. H. Colby, Polymer Physics (Oxford Univ. Press, New York, 2003).

20. D. C. Boris and R. H. Colby, Macromolecules 31, 5746 (1998).

21. A. V. Dobrynin, R. H. Colby, and M. Rubinstein, Macromolecules 28, 1859 (1995).

22. I. Suleimenov, D. Shaltykova, Z. Sedlakova, G. Mun, N. Semenyakin, D. Kaldybekov, and P. Obukhova, Appl. Mech. Mater. 467, 58 (2014).

23. Y. J. Bo, V. V. Khutoryanskiy, G. A. Mun, and Z. S. Nurkeeva, Polym. Sci., Ser. A 44, 1094 (2002).

24. G. M. Bartenev and L. A. Vishnitskaya, Vysokomol. Soedin. 6, 751 (1964).

25. G. S. Manning, J. Chem. Phys. 51, 924 (1969).

26. A. D. Antipina, V. Yu. Baranovskii, I. M. Papisov, and V. A. Kabanov, Vysokomol. Soedin., Ser. A 14, 941 (1972). 